\title{
Predicción de la violencia en la pareja perpetrada desde la personalidad oscura, doble moral y celos en mujeres adultas de CABA y GBA
}

\author{
Pablo Christian González Caino ${ }^{1}$, Leila Herrera ${ }^{2}$ y Santiago Resett ${ }^{3}$
}

\begin{abstract}
RESUMEN
La violencia es un tema de gran preocupación en la Argentina. Muy pocos estudios han evaluado la violencia perpetrada por parte de las mujeres adultas en pareja. De este modo, el presente estudio buscó predecir la violencia ejercida por mujeres a partir de la personalidad oscura, la doble moral y los celos. Para responder a los objetivos de esta investigación, se constituyó una muestra intencional de 214 mujeres de CABA y GBA ( $M_{\text {edad }}$ $=26.30 ; D E=7.40$ ). El 78\% manifestó estar de novia o comenzando una relación; el 18\%, estar casada; el 6\%, estar divorciada, pero en otra relación. Contestaron un cuestionario de violencia perpetrada hacia la pareja, uno de personalidad oscura, uno de doble moral, uno de los celos y preguntas demográficas. Los resultados indicaron que los puntajes más altos de violencia perpetrada fueron la psicológica y la económica. Regresiones en bloques predijeron una varianza del 34\% y 30\% para la violencia psicológica y económica, con la psicopatía y los celos conductuales como los predictores significativos. Un modelo estructural para predecir la violencia mostró un ajuste adecuado lo que sugiere que, nuevamente, la personalidad oscura y los celos son predictores significativos. Por otra parte, emergieron diferencias en los perfiles psicosociales de mujeres con alta y baja perpetración de la violencia. En la Discusión, se analizan las implicancias de estos hallazgos y se brindan sugerencias para futuros estudios.
\end{abstract}

Palabras clave: violencia, género, personalidad oscura, moral, celos, mujeres.

\section{Prediction of intimate partner violence perpetrated from the dark personality, double standards and jealousy in adult women from CABA and GBA}

\begin{abstract}
Violence is a matter of great concern in Argentina. Very few studies have evaluated violence perpetrated by adult women in a relationship. In this way, the present study sought to predict violence exerted by women based on dark personality, double standards and jealousy. Responding to the objectives of this study, an intentional sample of 214 women from CABA and GBA (mean age $=26.30$ years; $S D=7.40$ ) was constituted. $78 \%$ said that they were dating or starting a relationship, $18 \%$ were married and $6 \%$ were divorced, but in another relationship. They answered a questionnaire on violence perpetrated against the partner, one with a dark personality, one with double standards, one with jealousy, and demographic questions. The results indicated that the highest scores for violence perpetrated were psychological and economic. Block regressions predicted 34\% and 30\%
\end{abstract}

\footnotetext{
${ }^{1}$ Consejo Nacional de Investigaciones Científicas y Técnicas (CONICET), Universidad Argentina de la Empresa, Argentina; pablo.cg.caino@hotmail.com; https://orcid.org/ 0000-0003-2387-5777

2 Universidad Argentina de la Empresa, Argentina; herreraleilat@gmail.com; https://orcid.org/0000-00018683-3027

${ }^{3}$ Consejo Nacional de Investigaciones Científicas y Técnicas (CONICET), Universidad Argentina de la Empresa, Argentina; santiago_resett@hotmail.com; https://orcid.org/0000-0001-7337-0617
} 
variance for psychological and economic violence, with psychopathy and behavioral jealousy being the significant predictors. On the ones hand, a structural model for predicting violence showed an adequate fit suggesting that, again, dark personality and jealousy were the significant predictors. On the other hand, differences emerged in the psychosocial profiles of women with high and low perpetration of violence. In the Discussion, the implications of these findings are analyzed and suggestions for future studies are provided.

Keywords: violence, gender, dark personality, moral, jealousy, women.

La violencia en la pareja o Intimate Partner Violence (IPV) es una problemática social y de salud pública, con graves consecuencias a corto y largo plazo, tanto para la salud fisica como para la salud mental (Karakurt et al., 2016; Miller \& McCaw, 2019; Smith et al., 2018). Hay tres tipos de violencia que constituyen la violencia en la pareja: (i) abuso psicológico/emocional, (ii) físico y (iii) sexual. El abuso psicológico/emocional refiere a los casos en que el perpetrador actúa de manera ofensiva o degradante hacia su pareja, la mayoria de las veces de manera verbal, incluyendo amenazas, burlas y/o restricciones (Capaldi \& Langhinrichsen-Rohling, 2012; Capaldi, Tiberio, Shortt, Low, \& Owen, 2020). La violencia física apunta al contacto físico forzado que ocurre entre parejas. Esto puede incluir empujones y bofetadas ligeras, así como actos más serios como golpes y violencia letal. Ambos tipos de violencia pueden causar grandes problemas para la víctima en varias esferas de su vida (Miller $\&$ McCaw, 2019). La violencia sexual se entiende a toda acción que obliga a una persona a mantener interacciones sexuales con el agresor o un tercero mediante el uso de la fuerza física, chantaje, soborno, manipulación o cualquier otra conducta (Bogante Rojas, 2008). Estadísticas de la Organización de las Naciones Unidas (2020) señalan que el 35\% de las mujeres ha experimentado violencia de pareja física y/o sexual en algún momento de su vida. Por otro lado, el Departamento de Justicia de los Estados Unidos observa que las mujeres de entre 16 y 24 años tienen mayor riesgo de padecer violencia de pareja no fatal, mientras que las mujeres de entre 24 y 29 años tienen mayor riesgo de ser asesinadas por sus parejas (Amar \& Gennaro, 2005). El género se conside un factor de riesgo importante en la violencia de pareja. La investigación sobre cómo el género impacta en el riesgo es, sin embargo, mixta (Daigle, Scherer, Fisher, \& Azimi, 2016). Según estadísticas del Ministerio de las Mujeres, Géneros y Diversidad (2020), se recibieron un total de 99156 denuncias sobre violencia de género en el 2020, siendo en el $83 \%$ de los casos una pareja amorosa o una expareja el agresor, mientras que el 63\% de las denunciantes eran mujeres de entre 15 y 44 años.

Históricamente, cuando la violencia en la pareja se reconoció como un problema en los Estados Unidos en la década de 1970, se hizo por preocupación específicamente por las mujeres. Nacido del movimiento de derechos de las mujeres, se llamó la atención sobre la difícil situación de aquellas mujeres que estaban en relaciones abusivas en las que los hombres eran los únicos agresores. El hecho de que los hombres también podrian ser maltratados por sus parejas no fue reconocido. Con el desarrollo de encuestas de autoinforme, como la Conflict Tactics Scale (Straus, 2007), comenzó a surgir una imagen de violencia de pareja intima que incluye tanto a hombres como a mujeres como víctimas y perpetradores. En la actualidad, el grado en el que los hombres y 
mujeres se involucran y experimentan la violencia en la pareja es objeto de acalorados debates (Daigle et al., 2016). La investigación ha destacado la elevada frecuencia de estas conductas y ha determinado una naturaleza bidireccional de las agresiones, lo que la convierte en una dimensión crítica de estudio debido a las controversias que incita (Capaldi \& LanghinrichsenRohling, 2012; Desmarais, Reeves, Nicholls, Telford, \& Fiebert, 2012a, 2012b).

En las últimas décadas, se ha publicado un número creciente de estudios que respaldan la afirmación de que las mujeres perpetran violencia a tasas similares que los hombres en cuanto a violencia física o psicológica, aunque generalmente, las mujeres recurren a la violencia en situaciones de defensa. A su vez, los hombres tienen tasas más altas de violencia sexual y control coercitivo que las mujeres (Swan et al., 2008). Por ejemplo, Straus y Ramírez (2007) realizaron un estudio en cuatro universidades y reportaron una ligera diferencia de género: el 35\% de las mujeres y el 30\% de hombres habían cometido violencia de pareja durante los últimos 12 meses. La perpetración también se ha comparado en hombres y mujeres de todo el mundo. En un estudio de 31 naciones (Asia y Medio Oriente, Australia y Nueva Zelanda, Europa, América Latina y América del Norte), Straus (2007) halló que las mujeres perpetraron violencia a tasas más altas en 21 de los 31 sitios. Los hallazgos son relativamente consistentes entre las citas, la convivencia y los matrimonios en muestras comunitarias. Sin embargo, algunos estudios sugieren que los participantes menores de 30 años evidencian tasas más altas de agresión particularmente por parte de las mujeres. Graña Gómez y Cuenca Montesino (2014) evaluaron el comportamiento agresivo en una muestra de 3578 parejas y los resultados reafirmaron la naturaleza bidireccional de la agresión psicológica y física.

La Tríada Oscura de la Personalidad comprende tres rasgos socialmente aversivos - maquiavelismo, narcisismo y psicopatía- que han sido estudiados como una constelación (Jones \& Paulhus, 2014, 2017; Miller, Vize, Crowe, \& Lynam, 2019), debido a su naturaleza socialmente indeseable y a los comportamientos fenotípicos que observa, como por ejemplo, la manipulación y sus similitudes conceptuales. Es factible identificar estos rasgos en las relaciones en las que los individuos pueden parecer encantadores al principio, pero que en las interacciones posteriores demuestran su "toxicidad", dado que tienden a emplear comportamientos en beneficio propio y de explotación interpersonal (Rauthmann, 2012).

El maquiavelismo se refiere a estrategias interpersonales que defienden el interés propio, el engaño y la manipulación (Jakobwitz \& Egan, 2006), y enlaza la tendencia a mantener creencias cínicas, frias, pragmáticas e inmorales con un afecto desapegado hacia los otros (Rauthmann \& Will, 2011). El trastorno narcisista de la personalidad ha sido definido por el DSM-III (American Psychiatric Association, 1980). Raskin y Hall (1981) perfilaron esta definición y elaboraron la versión subclínica del mismo mediante la construcción del Inventario Narcisista de la Personalidad (NPI). El modelo comprende que las conductas del trastorno narcisista de la personalidad son formas extremas que en individuos que no lo padecen se manifiestan en medidas normales. Las personas narcisistas son aquellas que tienen una tendencia hacia la grandiosidad, el exhibicionismo, tienden a defenderse ante las críticas, sus relaciones interpersonales se basan en la explotación del otro, en la falta de empatía y la pretensión. La psicopatía consta de dos elementos claves: el déficit de afecto (insensibilidad) y el déficit de autocontrol (impulsividad). Los 
psicópatas manifiestan su insensibilidad a corto plazo (Jones \& Paulhus, 2011; Visser, Bay, Cook, \& Myburgh, 2012). La Triada Oscura se ha utilizado para investigar el comportamiento antisocial en poblaciones subclínicas y proporcionan un valor adicional en la predicción de resultados antisociales, como la desconexión moral que justifica el comportamiento poco ético, la violencia comunitaria y la proclividad al acoso sexual (Egan, Hughes, \& Palmer, 2015; Pailing, Boon, \& Egan, 2014; Zeigler-Hill, Besser, Morag, \& Campbell, 2016). En el caso de la violencia de pareja, se ha encontrado que la psicopatía y el maquiavelismo son predictores de la violencia tanto física como psicológica, a la vez que predicen respuestas destructivas frente al conflicto y al control de la pareja (Carton \& Egan, 2017; Brewer et al., 2018). Por otro lado, la psicopatía tiene un efecto en la violencia perpetrada, tanto para hombres como para las mujeres (Kiire, 2017).

Ha habido un considerable interés en el tema del Doble Estándar Sexual desde su concepción (Reiss, 1960) que, en un principio, les permitía a los hombres doble estándar en relaciones sexuales prematrimoniales, mientras que no sucedia lo mismo con las mujeres. Este estándar consiste en evaluar a hombres y mujeres de distinta manera en situaciones similares utilizando diferentes criterios para ambos grupos (Álvarez-Muelas, Gómez-Barrocal, \& Sierra, 2020; Marks, Young, \& Zaikman, 2018). Aquellos individuos que apoyan este estándar suponen una mayor libertad sexual para los hombres que para las mujeres en determinados contextos y en relación a determinados comportamientos sexuales como por ejemplo, sexo antes del matrimonio, mantener relaciones sexuales con múltiples parejas, relaciones sexuales a temprana edad, relaciones sexuales casuales o sin compromiso, o desempeñar un rol activo en el sexo. En contraparte, una menor adhesión al doble estándar supone una mayor aceptación de la igualdad entre ambos sexos (García-Cueto et al., 2015; Sierra, Rojas, Ortega, \& Martín Ortiz, 2007). En los últimos años, las relaciones prematrimoniales y las relaciones sexuales fuera de una relación estable son más aceptables para ambos sexos, aún existen facetas de la sexualidad que siguen siendo valoradas de distinta forma en hombres y en las mujeres (Bordini \& Sperb, 2013). La doble moral sexual tiene múltiples formas de manifestaciones y, a la vez, es una variable predictora de los comportamientos violentos (Moyano, Monje, \& Sierra, 2017). Sin embargo, estudios recientes han demostrado que a pesar de que la doble moral sexual es más frecuente en hombres, las mujeres también presentan este tipo de actitudes (Farvid, Braun, \& Rowney, 2017).

Los celos románticos son una emoción compleja que se activan ante una amenaza real o percibida dentro de una relación sentimental. Estas amenazas son generadas por la percepción de una atracción real o potencial entre la pareja y un rival. Los celos son un estado que, según el contexto, pueden despertar emociones como tristeza (pérdida), ira (traición), miedo o ansiedad (Hart \& Legerstee, 2013; Martínez-León, Mathes, Avendaño, Peña, \& Sierra, 2017). Las investigaciones han proporcionado información valiosa sobre las operaciones fundamentales de las relaciones románticas (Holden, Zeigler-Hill, Pham, \& Shackelford, 2014). Se ha encontrado que los comportamientos de retención de pareja están asociados con la violencia hacia una pareja (Kaighobadi, Shackelford, \& Goetz, 2009), la frecuencia de las relaciones sexuales (Kaighobadi \& Shackelford, 2008) y los estímulos negativos intersexuales, es decir, la inducción de celos, la manipulación emocional y de compromiso, la derogación de posibles competidores y el castigo de la amenaza de infidelidad 
de un compañero (de Miguel \& Buss, 2011; Shackelford, Goetz, Buss, Euler, \& Hoier, 2005). Los hallazgos anteriores sugieren que estos rasgos pueden estar asociados con las dimensiones conductuales, cognitivas y emocionales de los celos románticos. Es importante destacar que los celos románticos se han relacionado positivamente con sentimientos de inseguridad, baja autoestima y autoconciencia (Khanchandani \& Durham, 2009). Los celos desadaptativos pueden volverse problemáticos cuando se presentan en formas de inseguridad, violencia, manipulación, autoconciencia, ansiedad y otras formas desventajosas (Wade \& Walsh, 2008). Los celos y las conductas controladoras son variables consideradas como facilitadoras de la violencia en la pareja ya que estas aumentan el riesgo de agredir (Fernández-Fuertes \& Fuertes, 2010). En la Argentina, en el $41 \%$ de los casos de violencia doméstica grave en el período 2018-2020, los informantes señalaron que las situaciones de celos por parte del agresor eran el principal detonante (Oficina de Violencia Doméstica, 2020b).

En cuanto a diferencias de género, la hipótesis evolutiva establece que los hombres pueden experimentar más celos en casos de infidelidad sexual, y las mujeres tienden a experimentarlos en casos de infidelidad emocional (Bendixen, Kennair, \& Buss 2015). Existe una fuerte asociación entre la violencia (física y verbal) y los celos (Kar \& O’Leary, 2013). Estos últimos se identifican como dos de los mediadores más importantes para aumentar la presencia de celos mórbidos y delirantes (Echeburúa \& Amor, 2016), y quienes padecen esta condición tienen un mayor número de intentos de asesinato contra su pareja (Easton \& Shackelford, 2009).

Entre 2003-2012 la National Crime Victimization Survey (NCVS) indicó que 1.4 por 1000 hombres sufrió violencia fisica por parte de su pareja; los hombres representaron un $24 \%$ de víctimas de violencia en la pareja (Truman $\&$ Morgan, 2014). Sin embargo, la mayoría de las personas no consideran que la violencia perpetrada contra ellos sea criminal, particularmente si es por parte de una mujer. En la última década, la violencia de género ha adquirido una mayor notoriedad a partir de los movimientos activistas de organizaciones feministas y organizaciones civiles. A nivel internacional, en Naciones Unidas se ha propuesto como objetivo para el 2030 la erradicación de todas las formas de discriminación hacia las mujeres. El Estado argentino ha suscripto a tratados y convenciones para demostrar su compromiso ante esta problemática; desde el año 2012 se lleva un registro único de casos de violencia contra las mujeres a nivel nacional (Instituto Nacional de Estadística y Censos, 2019). Existen gran variedad de estudios sobre violencia en donde la mujer es víctima del hombre. Sin embargo, la violencia en la pareja no es un fenómeno unidireccional. Más de 200 estudios respaldan que la bidireccionalidad de la violencia en la pareja se da hasta en un $80 \%$ de las relaciones violentas (Langhinrichsen-Rohling, Misra, Selwyn, \& Rohling, 2012; Straus, 2006). Actualmente en Argentina no existen programas para asistir a hombres que padecen violencia en la pareja. El único indicador hasta el momento se obtiene de la Oficina de Violencia Doméstica (OVD) creada en 2006 por la Corte Suprema de Justicia de la Nación: en el primer trimestre del 2020, el 50\% de las denuncias realizadas fueron por violencia en la pareja, de las que el 14\% son mujeres que fueron denunciadas por sus parejas (Oficina de Violencia Doméstica, 2020a).

Aunque el tema de la violencia de pareja está sumamente estudiado, pocas investigaciones se han llevado a cabo en las naciones de América Latina, como la Argentina, con poblaciones de mujeres que llevan a cabo dichas conductas. Por otra parte, la presente investigación empleó un modelo de 
ecuaciones estructurales para su predicción, en lugar de usar solamente regresiones lineales. El modelo elegido presenta la ventaja de permitir un análisis más complejo de las relaciones entre las variables, como la incorporación de constructos latentes en dicho análisis (Kline, 2015).

\section{Objetivos}

Por todo lo expuesto anteriormente, el presente trabajo tuvo como objetivos: (1) describir los niveles de violencia perpetrada, tanto psicológica como económica, la personalidad oscura, la doble moral y los celos en mujeres adultas en pareja de la Ciudad de Buenos Aires y del Gran Buenos Aires, Argentina; (2) predecir la violencia perpetrada a partir de la personalidad oscura, la doble moral y los celos; (3) poner a prueba un modelo de ecuaciones estructurales para predecir la violencia perpetrada a partir de dichas variables; (4) analizar las diferencias de personalidad oscura, la doble moral y los celos entre perfiles de alta y baja perpetración de violencia.

\section{MÉTODO}

\section{Participantes}

Para responder a los objetivos del presente estudio, se constituyó una muestra intencional de 214 mujeres heterosexuales de entre 18 y 40 años, con una media de edad de 26.30 años $(D E=7.40)$, de la Ciudad Autónoma de Buenos Aires y del Gran Buenos Aires, Argentina. El 78\% manifestó estar de novia; el 18\%, estar casada; el $6 \%$, estar divorciada, pero en otra relación. A su vez, el $41 \%$ de las participantes manifestó estar en una relación hace menos de 3 años; el 23\%, desde hace entre 3 y 5 años; y el 36\%, hace más de 5 años. Por último, el 46\% manifestó vivir todavía con sus padres; el $42 \%$, con su pareja; el $12 \%$ restante, vivir solo. Los criterios de inclusión fueron: ser heterosexual, ser mujer $-\mathrm{O}$ autopercibirse como tal-, tener entre 18 y 40 años, residir en la Ciudad Autónoma de Buenos Aires o en el Gran Buenos Aires, y estar en pareja actualmente.

\section{Instrumentos}

Cuestionario sociodemográfico. Se recabaron datos tales como edad, residencia, estado civil, años de estar en pareja, etc.

Cuestionario de Violencia en la Pareja (Cienfuegos-Martínez, 2014). Permite evaluar tanto la violencia recibida como la ejercida en la situación de vida en la pareja, ya sea matrimonio, convivencia o noviazgo. En el presente trabajo, se usaron las dimensiones sobre la violencia ejercida hacia la pareja. El rango de respuesta es de cinco puntos - 1 (nunca) a 5 (siempre)- La escala de violencia ejercida contra la pareja se compone de 11 items y posee tres dimensiones: violencia psicológica/física con siete îtems, violencia económica/social con tres ítems, y violencia sexual con un ítem. Ejemplos de estos son: "He llegado a insultar a mi pareja", "Me he enojado cuando mi pareja me contradice o no está de acuerdo conmigo" de la dimensión de violencia psicológica/física, "Me molesta que mi pareja gaste su propio dinero" de la escala de violencia económica, y "No tomo en cuenta las necesidades sexuales de mi pareja" para la dimensión de sexualidad. Ambas escalas muestran índices adecuados de validez y confiabilidad. La escala de violencia ejercida explica un $61.8 \%$ de la varianza con un Alfa de Cronbach de .84 (Cienfuegos-Martínez, 2014). El factor de violencia psicológica/física alude a gritos, insultos y negligencia a través de 
los cuales una persona busca dañar a su pareja psicológicamente, utilizando la violencia si fuera necesario para lograr que haga lo que él/ella quiere. La violencia económica contempla el control del perpetrador sobre su pareja al limitar el acceso al dinero. Se incluye un indicador de violencia sexual que hace referencia a la negligencia sexual. En el presente estudio, para la violencia ejercida (perpetrador) se reportaron a de .82 y .54.

Escala Breve de Triada Oscura de la Personalidad (SD3; Jones \& Paulhus, 2014). El SD3 es una escala breve de autoinforme que cuenta con 27 items agrupados en tres subescalas. Estas últimas evalúan las tres dimensiones de la personalidad oscura de forma conjunta -narcisismo, maquiavelismo $\mathrm{y}$ psicopatía- de forma subclínica. Consta de nueve items para cada dimensión, por ejemplo: "No es bueno o prudente contar tus secretos", "Me gusta manipular o engañar a las personas para lograr lo que quiero", "La gente me ve como un líder nato", "Hay cosas que conviene ocultar y que los demás no necesitan saber". La opción de respuesta se encuentra en el formato de tipo likert con cinco opciones (desde 1 totalmente en desacuerdo a 5 totalmente de acuerdo). Para el presente estudio, se utilizó la versión de Nieto Ribes (2015), con buenas propiedades psicométricas en población española. A su vez, en el presente estudio los a de Cronbach fueron de .69 para la dimensión maquiavelismo, .71 para psicopatía y .63 para narcisismo.

Double Standard Scale (DSS; Caron, Davis, Halteman, \& Stickle, 1993). Es de las escalas de autoinforme más utilizadas para evaluar la Doble Moral Sexual. Con una estructura unidimensional, está formada por nueve ítems contestados en una escala tipo likert de cinco puntos (de 1 totalmente en desacuerdo a 5 totalmente de acuerdo) tales como: "Se espera que una mujer sea menos experimentada sexualmente que su pareja", "Es importante que un hombre tenga múltiples encuentros sexuales para ganar experiencia", "Es peor que una mujer sea promiscua, a que lo sea un hombre", "Es decisión del hombre comenzar el sexo". A mayores niveles de puntuación hay una mayor presencia de doble moral sexual. Caron et al. (1993) reportaron una adecuada fiabilidad en su consistencia interna, así como adecuados indicadores de validez. Para el presente estudio, se utilizó la versión adaptada al español der Sierra, Monge, Santos-Iglesias y Aparicio (2010) que presentó buenas propiedades psicométricas en población española. El a de Cronbach para el presente estudio fue de .78 .

Multidimensional Jealousy Scale (MJS; Pfeiffer \& Wong, 1989). Está constituida por 24 items que representan tres dimensiones: celos cognitivos, compuesta por ocho ítems ("Sospecho que mi pareja está viendo en secreto a alguien del sexo opuesto", "Sospecho que mi pareja puede tener intimidad fisica con otro miembro del sexo opuesto detrás de mis espaldas"); celos conductuales con ocho ítems ("Reviso los cajones, bolsos o bolsillos de mi pareja", "Llamo a mi pareja inesperadamente, solo para ver si él / ella está allî"); los celos afectivos con ocho ítems ("Mi pareja me comenta lo bien que se ve un miembro del sexo opuesto en particular", "Me muestra mucho interés o emoción al hablar con alguien del sexo opuesto"). Posee una escala likert de siete puntos (de 1 nunca a 7 todo el tiempo). El MJS resulta útil para proporcionar una imagen clara de las relaciones entre los componentes de los celos y diversas variables psicológicas. La escala presenta una alta consistencia interna y una adecuada validez, con una varianza explicada del $62 \%$ y, en todos los casos, sus a de Cronbach para cada dimensión resulta ser superior a .70 (Pfeiffer \& Wong, 1989). Para esta investigación, se realizó una traducción a la inversa por 
expertos de esta escala, y se la comparó con la versión original para comprobar su equivalencia. Los a de Cronbach fueron $.90, .87$ y .81 respectivamente para la presente muestra.

\section{Procedimiento de recolección de datos}

Todas las participantes fueron contactadas a través de la herramienta Google Forms y a través de las redes sociales Facebook, Instagram, Twitter y Whatsapp. Primero, se verificaba la situación en pareja de la participante antes de poder contestar la batería de instrumentos. El link incluía la explicación del estudio, el consentimiento informando - de lo contrario la batería no se podía completar-y los tests. A su vez, antes de comenzar, se les informaba sobre la confidencialidad, el anonimato y los fines académicos de la investigación.

\section{Procedimientos de análisis de datos}

Todos los análisis estadísticos tanto descriptivos (medias y desvíos) como inferenciales (regresión múltiple por bloques y comparaciones de medias) fueron realizados con el software SPSS 23. En el caso de la regresión múltiple por bloques, se colocaron los puntajes de personalidad oscura, mientras que los de doble moral y celos se colocaron en un segundo bloque. A su vez, para la realización de los modelos estructurales se utilizó el software AMOS 21, utilizando el método de máxima verosimilitud.

Los datos de las variables estudiadas tenían distribución normal: de -1.20 a 1.87 de asimetría, y -0.96 a 3.66 de curtosis en el caso de la personalidad oscura; de 1.20 a 2.39 de asimetría, y 0.60 a 4.38 de curtosis para la doble moral; -2.73 a 4.20 de asimetría, y de -0.78 a 18.75 de curtosis en el caso de los celos; de 0.53 a 6.21 de asimetría, y de 0.42 a 51.20 de curtosis en el caso de la perpetración de violencia. Valores de asimetría mayores de 3 y de curtosis de 8 a 20 son considerados extremos (Kline, 2015; Tabacknick \& Fidell, 2014), por lo que se optó por utilizar el método de Máxima verosimilitud robusto para evaluar el ajuste del modelo propuesto. Los indicadores de ajuste que se tuvieron en cuenta fueron el CFI (Comparative fix index), el TLI (Tucker-Lewis Index) que deben ser por arriba de .90, y el RMSEA (Root mean square error of approximation) que debe estar debajo de .10 para ser aceptable (Bentler, 1992; Byrne, 2010) para medir el error del modelo, recomendado por Hooper, Coughlan y Mullen (2008).

Por último, se realizó un análisis de clusters bietápico con los puntajes en violencia económica y psicológica para determinar las diferencias entre grupos de alta y baja perpetración de violencia sobre la personalidad oscura, la doble moral y los celos cognitivos.

\section{RESULTADOS}

Con respecto al primer objetivo, la Tabla 1 muestra los puntajes medios y desvíos para violencia, donde el puntaje mayor entre las participantes fue el de violencia psicológica perpetrada.

En el caso de la personalidad oscura y sus respectivas dimensiones, se encontró la psicopatía con el puntaje mayor como se puede observar en la Tabla 2. A su vez, en la Tabla 3 se encuentran los puntajes y desvíos para las variables de doble moral y celos con un puntaje medio de $12.30(D E=4.17)$ para la doble moral, y a los celos cognitivos con el mayor puntaje. 
Tabla 1.

Medias y desvíos típicos de los puntajes violencia en mujeres adultas de CABA y GBA $(n=214)$.

\begin{tabular}{lcc}
\hline & $\boldsymbol{M}$ & $\boldsymbol{D E}$ \\
\cline { 2 - 3 } Violencia psicológica & 10.88 & 3.07 \\
Violencia económica & 3.46 & 0.91 \\
\hline
\end{tabular}

Tabla 2.

Medias y desvíos típicos de los puntajes de personalidad oscura mujeres adultas de CABA y GBA $(n=214)$.

\begin{tabular}{lcc}
\hline & $\boldsymbol{M}$ & $\boldsymbol{D E}$ \\
\cline { 2 - 3 } Maquiavelismo & 19.19 & 4.56 \\
Narcisismo & 16.34 & 3.03 \\
Psicopatía & 21.57 & 4.78 \\
\hline
\end{tabular}

Tabla 3.

Medias y desvíos típicos de los puntajes de doble moral y celos mujeres adultas de CABA y GBA $(n=214)$.

\begin{tabular}{lcc}
\hline & $\boldsymbol{M}$ & $\boldsymbol{D E}$ \\
\cline { 2 - 3 } Doble moral & 12.30 & 4.17 \\
Celos cognitivos & 45.05 & 10.18 \\
Celos emocionales & 42.39 & 8.64 \\
Celos conductuales & 15.90 & 7.47 \\
\hline
\end{tabular}

Con respecto al segundo objetivo, el análisis de regresión múltiple por bloques para la perpetración de violencia psicológica mostró un modelo significativo $(p<.001)$, en el que el primer bloque explicó un $15 \%$ de la varianza para luego subir a $34 \%$ al agregarse el segundo bloque (Tabla 4).

Tabla 4.

Predicción de la violencia psicológica perpetrada por mujeres adultas de CABA y GBA.

\begin{tabular}{|c|c|c|c|}
\hline & & Bloque 1 & Bloque 2 \\
\hline \multirow{2}{*}{ Maquiavelismo } & $t$ & -0.09 & -0.57 \\
\hline & $\beta$ & -0.01 & -0.04 \\
\hline \multirow{2}{*}{ Narcisismo } & $t$ & -1.03 & -1.02 \\
\hline & $\beta$ & -0.07 & -0.07 \\
\hline \multirow{2}{*}{ Psicopatía } & $t$ & $5.23^{* *}$ & $3.72^{* \star}$ \\
\hline & $\beta$ & 0.42 & 0.28 \\
\hline \multirow{2}{*}{ Doble moral } & $t$ & - & -0.34 \\
\hline & $\beta$ & & -0.02 \\
\hline \multirow{2}{*}{ Celos cognitivos } & $t$ & - & -1.63 \\
\hline & $\beta$ & & -0.11 \\
\hline \multirow{2}{*}{ Celos emocionales } & $t$ & - & 0.17 \\
\hline & $\beta$ & & 0.01 \\
\hline \multirow{2}{*}{ Celos conductuales } & $t$ & - & $6.34^{* *}$ \\
\hline & $\beta$ & & 0.41 \\
\hline$\overline{R^{2}}$ & & $15 \%$ & $34 \%$ \\
\hline
\end{tabular}


En el caso de la perpetración de violencia económica, como se puede observar en la Tabla 5, también se encontró un modelo estadísticamente significativo $(p<.001)$, donde el primer bloque explicó un $7 \%$ de la varianza y un $30 \%$ cuando se sumaba el segundo bloque. Nuevamente, los mayores predictores fueron la psicopatía $(t=2.09, \beta=0.16, p<.05)$ y los celos conductuales $(t=8.07, \beta=0.53, p<.001)$.

Tabla 5.

Predicción de la violencia económica perpetrada por mujeres adultas de CABA y $G B A$.

\begin{tabular}{|c|c|c|c|}
\hline & & Bloque 1 & Bloque 2 \\
\hline \multirow{2}{*}{ Maquiavelismo } & $t$ & -0.34 & -0.36 \\
\hline & $\beta$ & -0.03 & -0.03 \\
\hline \multirow{2}{*}{ Narcisismo } & $t$ & 0.59 & 0.04 \\
\hline & $\beta$ & 0.04 & 0.00 \\
\hline \multirow{2}{*}{ Psicopatía } & $t$ & $3.28^{* *}$ & $2.09^{*}$ \\
\hline & $\beta$ & 0.27 & 0.16 \\
\hline \multirow{2}{*}{ Doble moral } & $t$ & - & -1.09 \\
\hline & $\beta$ & & -0.07 \\
\hline \multirow{2}{*}{ Celos cognitivos } & $t$ & - & 1.25 \\
\hline & $\beta$ & & 0.08 \\
\hline \multirow{2}{*}{ Celos emocionales } & $t$ & - & -0.81 \\
\hline & $\beta$ & & -0.05 \\
\hline \multirow{2}{*}{ Celos conductuales } & $t$ & - & $8.07^{\star \star}$ \\
\hline & $\beta$ & & 0.53 \\
\hline$\overline{R^{2}}$ & & $7 \%$ & $30 \%$ \\
\hline
\end{tabular}

Para analizar los efectos de la personalidad oscura, los celos y la doble moral sobre la perpetración de violencia, tanto psicológica como económica, se realizaron dos modelos de ecuaciones estructurales sin restricciones y con restricciones de los efectos indirectos. En la Figura 1, se puede observar el modelo sin restricciones propuesto, colocando la personalidad oscura, los celos y la doble moral como variables observables, y a la violencia perpetrada como variable endógena. Se encontró un ajuste aceptable $(C F I=.93, T L I=.88, R M S E A$ $\left.=.08 ; \chi^{2}(23)=54.87, p<.001\right)$. A su vez, en la Tabla 6 se muestran los predictores para la violencia perpetrada, donde se encontró un efecto directo de la personalidad oscura sobre la doble moral, y de los celos sobre la violencia perpetrada. 


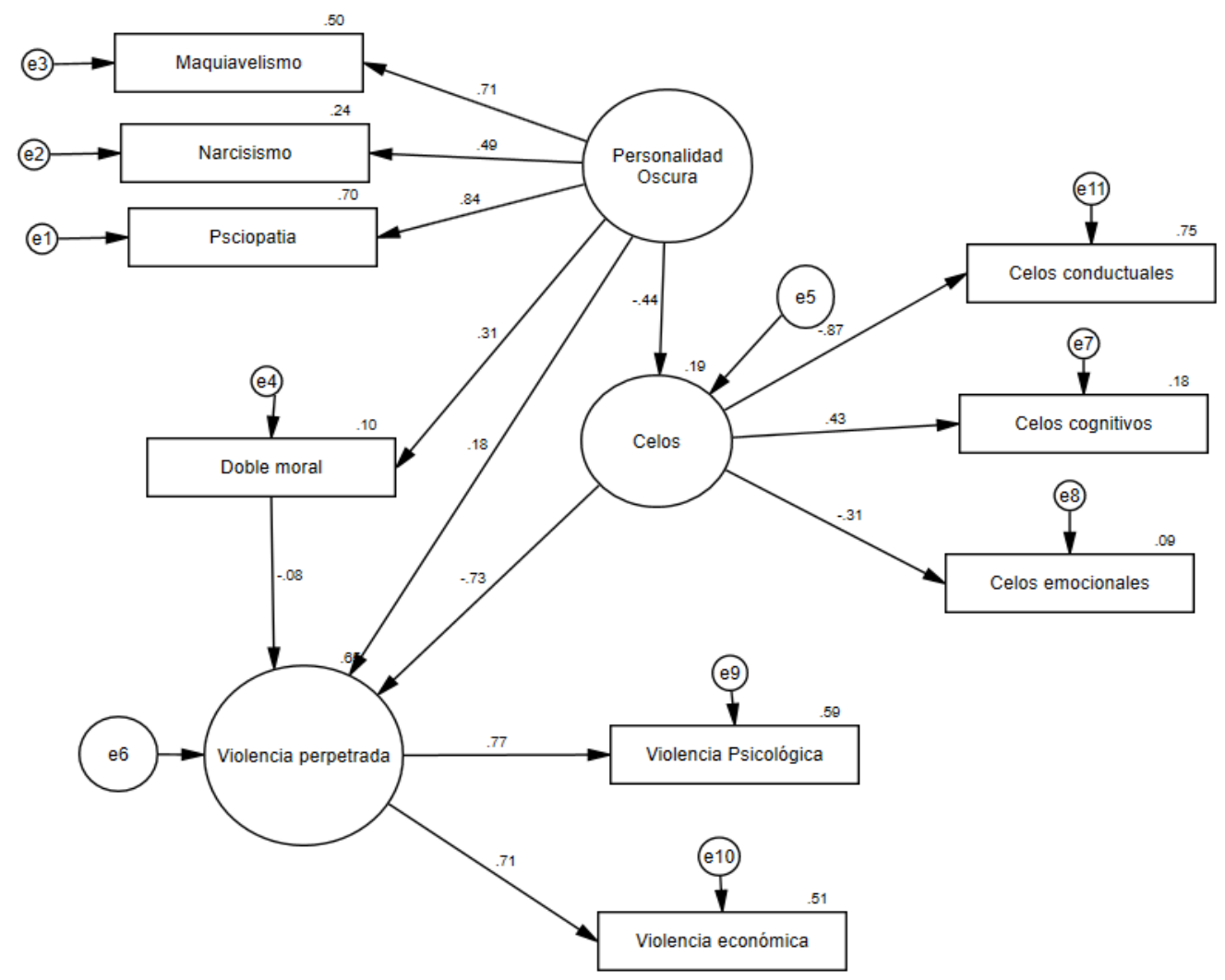

Figura 1. Modelo híbrido de ajuste con personalidad oscura, celos y doble moral como variables observables y la violencia perpetrada como variable endógena.

Tabla 6 .

Significatividad de los predictores del modelo híbrido de ajuste con personalidad oscura, celos y doble moral como variables observables y la violencia perpetrada como variable endógena.

\begin{tabular}{lc}
\hline Predictores & \\
Personalidad oscura $\rightarrow$ Doble Moral & $.31^{* *}$ \\
Personalidad oscura $\rightarrow$ Celos & $-.44^{* *}$ \\
Personalidad oscura $\rightarrow$ Violencia perpetrada & .18 \\
Doble Moral $\rightarrow$ Violencia perpetrada & -.08 \\
Celos $\rightarrow$ Violencia perpetrada & $-.73^{* *}$ \\
Personalidad oscura $\rightarrow$ Maquiavelismo & .71 \\
Personalidad oscura $\rightarrow$ Narcisismo & $.49^{* *}$ \\
Personalidad oscura $\rightarrow$ Psicopatía & $.84^{* *}$ \\
Celos $\rightarrow$ Celos cognitivos & .42 \\
Celos $\rightarrow$ Celos emocionales & $-.31^{* *}$ \\
Celos $\rightarrow$ Celos conductuales & $-.87^{* *}$ \\
Violencia perpetrada $\rightarrow$ Violencia psicológica & .77 \\
Violencia perpetrada $\rightarrow$ Violencia económica & $.71^{* *}$ \\
\hline$* p<01$ &
\end{tabular}

Por último, se llevó a cabo el análisis de cluster bietápico que permitió obtener dos grupos: uno de $n=180$ y otro de $n=34$. El primero se denominó bajos niveles de perpetración de violencia; el segundo, de niveles altos de violencia. Al comparar ambos grupos mediante la prueba $t$ de Student, con la pertenencia los conglomerados como factor entre sujetos, el análisis arrojó diferencias en psicopatía y celos conductuales en favor del grupo con alta 
perpetración de violencia, como se muestra en la Tabla 7. Por otro lado, el grupo con baja perpetración de violencia mostró un puntaje mayor en celos cognitivos. A su vez, se encontró una diferencia marginal en la doble moral $(t(212)=-1.81$, $p<.07)$, en favor del grupo de alta perpetración de violencia $(M=13.72, D E=$ 4.42) en comparación con el de baja perpetración $(M=12.12, D E=4.11)$.

Tabla 7.

Diferencias en puntaje de personalidad oscura, la doble moral y los celos en grupos con alta y baja perpetración de violencia en adultas de CABA y GBA.

\begin{tabular}{lcccccc}
\hline & \multicolumn{4}{c}{ Grupo } \\
\cline { 2 - 5 } & \multicolumn{2}{c}{$\begin{array}{c}\text { Baja } \\
n=180\end{array}$} & \multicolumn{2}{c}{$\begin{array}{c}\text { Alta } \\
n=34\end{array}$} & \multirow{2}{*}{$\begin{array}{c}\text { Prueba } \boldsymbol{t} \\
(\mathrm{gl}=212)\end{array}$} \\
\cline { 2 - 5 } & $\boldsymbol{M}$ & $\boldsymbol{D E}$ & $\boldsymbol{M}$ & $\boldsymbol{D E}$ & \\
\cline { 2 - 5 } Maquiavelismo & 18.95 & 4.42 & 21.00 & 5.29 & $-2.11^{*}$ \\
Narcisismo & 16.25 & 3.06 & 17.04 & 2.79 & -1.21 \\
Psicopatía & 20.98 & 4.38 & 26.00 & 5.48 & $-5.21^{* *}$ \\
Doble Moral & 12.12 & 4.11 & 13.72 & 4.42 & $-1.81^{+}$ \\
Celos cognitivos & 45.85 & 9.99 & 39.04 & 9.81 & $3.21^{* *}$ \\
Celos emocionales & 42.26 & 8.32 & 43.36 & 10.89 & -.59 \\
Celos conductuales & 14.64 & 6.02 & 25.44 & 10.24 & $-7.65^{* *}$ \\
\hline "P< & & & & &
\end{tabular}

${ }^{*} p<.05^{* *} p<.01{ }^{+} p<.07$

\section{DISCUSIÓN}

La violencia ejercida a la pareja es una conducta con importantes costos psicosociales y económicos. Sin embargo, en América Latina pocas investigaciones se han llevado a cabo sobre esta temática en mujeres adultas (Thomson, Kiehl, \& Bjork, 2019) ya que la mayoría de los estudios en el tema se han enfocado en varones adultos. Para este fin, se constituyó una muestra de 214 mujeres heterosexuales en pareja de entre 18 y 40 años de la Ciudad Autónoma de Buenos Aires y del Gran Buenos Aires, Argentina. Las participantes completaron un Cuestionario sociodemográfico, el Cuestionario de Violencia en la Pareja (Cienfuegos-Martínez, 2014), la Escala breve de triada oscura de la personalidad (SD3; Jones \& Paulhus, 2014), la Double Standard Scale (DSS; Caron et al., 1993) y la Multidimensional Jealousy Scale (MJS; Pfeiffer \& Wong, 1989).

Con respecto a los niveles de violencia, los resultados mostraron niveles más altos de violencia psicológica que económica. Esto no es sorprendente ya que la violencia psicológica es una de las formas más frecuente para las mujeres (Cienfuegos-Martínez, 2014) debido tanto a factores sociales como físicos, como por ejemplo, una menor masa corporal que los varones. Aunque pocos estudios han examinado la violencia psicológica o emocional en el mundo que ejercen las mujeres, las pocas investigaciones disponibles indican que las mujeres tienen la inclinación a perpetrar este tipo de violencia de forma individual o en combinación con otras formas (Williams, Ghandour, \& Kub, 2008). En otras investigaciones con muestras españolas también se detectaron mayores niveles de violencia psicológica en las mujeres que de otro tipo, como la física (Sebastián, Verdugo, \& Ortiz, 2014). Lamentablemente, el instrumento aquí usado solamente pregunta sobre dos tipos de violencia. Sin embargo, estos datos deben tomarse con cautela debido a la muestra de un tamaño no muy grande e intencional. 
Si bien ha habido cambios positivos en cuanto al deseo sexual de las mujeres en la sociedad, el doble estándar sexual sigue instalado en el pensamiento femenino e incluso con mayor prejuicio que en el de los hombres. Esto se debe a que el concepto de sexualidad activa y deseosa es considerado positivamente entre los hombres, pero denigrada y regulada por un etiquetado negativo con las mujeres. Las investigaciones entre la doble moral sexual y la Triada Oscura de la Personalidad son muy escasas. Algunas investigaciones sugieren que aquellas mujeres con niveles altos de personalidad oscura tienden a tener una mayor competencia sexual, lo que sugiere que tienen mayores herramientas para desenvolverse en el terreno sexual (Jonason, Webster, \& Schmitt 2009, Jonason \& Webster, 2010). Por lo tanto, es posible suponer que, a niveles más elevados de personalidad oscura, el doble estándar sexual será menor.

En lo relativo a la predicción de la violencia, se observó que la psicopatía y los celos conductuales eran los predictores significativos de la perpetración para la forma psicológica y económica. Se predecía un alto porcentaje de varianza con 34\% y 30\% respectivamente. Que la psicopatía sea un predictor significativo no es sorprendente ya que es uno de los predictores más estudiados al respecto (Thomson, Bozgunov, Psederska, \& Vassileva, 2019). La literatura científica destaca que las personas con rasgos psicopáticos son insensibles, poco empáticas e impulsivas lo que explicaría sus conductas violentas, como la perpetración de la violencia física (Jones \& Neria, 2015; Muris, Merckelbach, Otgaar, \& Meijer, 2017; Paulhus, Curtis, \& Jones, 2018). Lo interesante es que aquí la psicopatía está relacionada con la violencia emocional y económica. Esto se ha detectado también dentro del género femenino al igual que en el presente estudio (Thomson et al., 2019).

Sin embargo, en esta investigación se observó que la psicopatía no era el predictor más significativo, sino que sí lo eran los celos conductuales, tanto para la violencia emocional como para la económica. Algunos estudios señalan que altos niveles de celos pueden asociarse con conductas de agresividad y violencia (Fernández-Fuertes \& Fuertes, 2010). Por otra parte, que sean los celos conductuales los predictores significativos - no los emocionales ni los cognitivos - pondría en evidencia que la dimensión física que conlleva este tipo de comportamiento (acercarse físicamente a la pareja o hacerle una visita secreta) explicaría su significativa asociación con la violencia dentro del contexto de la pareja. Los celos, de este modo, son un robusto predictor de la violencia, más, incluso, que la psicopatía, lo que coincide con muchas investigaciones que indican que son un fuerte disparador de la violencia dentro de la pareja (Pichon et al. 2020). Futuros estudios deberían examinar si el alto valor predictor de los celos se da solamente en las mujeres o en ambos géneros.

Que la doble moral no se asocie con ningún tipo de violencia puede deberse a que la doble moral y el test aquí usado preguntaba sobre comportamientos que se relacionan más con un rol femenino tradicional: no ser promiscua, no tener la iniciativa en el sexo, etc. los cuales -probablemente-se relacionan más con sufrir violencia de la pareja más que ejercerla. Aunque más investigación es necesario a este respecto.

Finalmente, el modelo estructural con un ajuste adecuado indicó nuevamente que la personalidad oscura y los celos son predictores significativos de la perpetración de la violencia, mientras que la doble moral no lo es.

Recientemente, se han encontrado asociaciones positivas entre la psicopatía y las dimensiones cognitivas y conductuales del MJS. Esto se debe a 
la propensión de los psicópatas a la impulsividad temeraria, a la búsqueda de emociones fuertes $y$, en especial, a la indiferencia cruel que sugiere que la psicopatía estaría más relacionada con los celos conductuales, seguidos de los celos cognitivos. Sin embargo, no hay una relación significativa con los celos emocionales debido a una falta inherente de apego emocional a los demás (Chin, Atkinson, Raheb, Harris, \& Vernon, 2017). Las mujeres que poseen altos niveles de psicopatía apelan a la inducción de los celos románticos como un proceso de comportamiento estratégico para provocar una respuesta de celos reactiva de su pareja, para logar un objetivo que generalmente es la retención de esta (Jonason, Li \& Buss, 2010; Mattingly, Whitson, \& Mattingly, 2012). Las mujeres tienden a emplear los celos con mayor frecuencia que los hombres. En general, sus razones son para probar la relación y el deseo de poder o control sobre el otro. Asimismo, esta inducción de celos se asocia con la agresión y la necesidad de control sobre el otro. De este modo, al comparar los niveles de perpetración de mujeres con bajos niveles de violencia versus altos niveles de violencia, nuevamente las diferencias de puntajes más elevados se presentaron en psicopatía y en celos conductuales.

El presente trabajo tiene una serie de limitaciones que deben ser mencionadas. En primer lugar, la selección de la muestra intencional y de un tamaño pequeño. Además, es conocida la limitación inherente al uso de autoinformes; mucho más en variables tales como la violencia ejercida donde la falta de honestidad y/o la deseabilidad social pueden ejercer un rol distorsivo. Por otra parte, el test solamente evalúa violencia psicológica y económica, y deja de lado otras importantes como la sexual y la física, que también puede ser ejercida por las mujeres. Por otra parte, el cuestionario de Cienfuego Martínez (2014) tiene la limitación de no inquirir en un lapso de tiempo lo que genera que la frecuencia quede a criterio del participante a diferencia de otros tests como la CTS-2 (Straus Hamby, Boney-McCoy, \& Sugarman, 1996). También, el haber evaluado todas las variables con el autoinforme aumentan artificialmente las correlaciones entre las variables por la varianza compartida por el método de recolección de datos. Por otra parte, el tipo de estudio transversal no permite inferir direcciones de causalidad entre las variables.

Futuros estudios deberian examinar esta importante problemática en muestras de mayor tamaño y seleccionadas al azar de distintas regiones de la Argentina para poder generalizar los resultados. Sería interesante también trabajar con una muestra de adolescentes, de varones y con sujetos de distinta orientación sexual para observar si los predictores de la violencia ejercida son similares o no para dichas poblaciones. También sería útil examinar la violencia en poblaciones no comunitarias, como las mujeres privadas de la libertad. Asimismo, se debería examinar la violencia con otras técnicas de recolección de datos para evitar las limitaciones del autoinforme, como, por ejemplo, que el otro miembro de la pareja informe sobre el participante. Por otra parte, futuras investigaciones longitudinales serian capaces de determinar la estabilidad temporal de la violencia, así como evaluar las interrelaciones entre las distintas variables. Finalmente, sería deseable llevar a cabo acciones para prevenir la violencia de pareja.

\section{REFERENCIAS}

Álvarez-Muelas, A., Gómez-Berrocal, C., \& Sierra, J. C. (2020). Typologies of sexual double standard adherence in spanish population. European Journal of Psychology Applied to Legal Context, 13(1), 1-7. https://doi.org/10.5093/ejpalc2021a1

Amar, A. F. \& Gennaro, S. (2005). Dating violence in college women: Associated physical injury, healthcare 
usage, and mental health symptoms. Nursing research, 54(4), 235-242. https://doi.org/10.1097/00006199-200507000-00005

American Psychiatric Association (1980). Diagnostic and statistical manual of mental disorder (3 ${ }^{\mathrm{a}}$ ed.). Washington, DC: Asociación Americana de Psiquiatria.

Bendixen, M., Kennair, L. E. O., \& Buss, D. M. (2015). Jealousy: Evidence of strong sex differences using both forced choice and continuous measure paradigms. Personality and Individual Differences, 86, 212-216. https://doi.org/10.1016/j.paid.2015.05.035

Bentler, P. M. (1992). On the fit of models to covariances and methodology to the Bulletin. Psychological bulletin, 112(3), 400. https://doi.org/10.1037/0033-2909.112.3.400

Bogante Rojas, J. (2008). Violencia doméstica. Medicina Legal de Costa Rica, 25, 55-60.

Bordini, G. S. \& Sperb, T. M. (2013). Sexual double standard: A review of the literature between 2001 and 2010. Sexuality \& Culture, 17, 686-704. https://doi.org/10.1007/s12119-012-9163-0

Brewer, G., Bennett, C., Davidson, L., Ireen, A., Phipps, A. J., Stewart-Wilkes, D., \& Wilson, B. (2018). Dark triad traits and romantic relationship attachment, accommodation, and control. Personality and Individual Differences, 120, 202-208. https://doi.org/10.1016/j.paid.2017.09.008

Byrne, B. M. (2010). Structural equation modeling with AMOS Basic concepts, applications, and programming (Multivariate Applications Series). New York: Routledge/Taylor \& Francis Group.

Capaldi, D. M. \& Langhinrichsen-Rohling, J. (2012). Informing intimate partner violence prevention efforts: Dyadic, developmental, and contextual considerations. Prevention Science, 13(4), 323-328. https://doi.org/10.1007/s11121-012-0309-y

Capaldi, D. M., Tiberio, S. S., Shortt, J., Low, S., \& Owen, L. D. (2020). Associations of exposure to intimate partner violence and parent-to-child aggression with child competence and psychopathology symptoms in two generations. Child Abuse \& Neglect, 103, 104434. https://doi.org/10.1016/j.chiabu.2020.104434

Caron, S. L., Davis, C. M., Halteman, W. A., \& Stickle, M. A. R. L. A. (1993). Double standard scale. En T. D. Fisher, C. M. Davis, W. L. Yarber, \& S. L. Davis (Eds.) Handbook of sexuality-related measures (pp. 182183). UK: Routledge Handbooks Online. https://doi.org/10.1037/t57716-000

Carton, H. \& Egan, V. (2017). The dark triad and intimate partner violence. Personality and Individual Differences, 105, 84-88. https://doi.org/10.1016/j.paid.2016.09.040

Cienfuegos-Martínez, Y. (2014). Validación de dos escalas para evaluar violencia en la relación de pareja: Perpetrador/a y Receptor/a. Revista de Psicología Iberoamericana, 22(1), 62 -71.

Chin, K., Atkinson, B. E., Raheb, H., Harris, E., \& Vernon, P. A. (2017). The dark side of romantic jealousy. Personality and Individual Differences, 115, 23-29. https://doi.org/10.1016/j.paid.2016.10.003

Daigle, L. E., Scherer, H., Fisher, B. S., \& Azimi, A. (2016). Intimate Partner Violence Among College Students. En C. A. Cuevas \& C. M. Rennison (Eds.), The Wiley Handbook on the Psychology of Violence (pp. 371395). New Jersey: John Wiley \& Sons, Inc. https://doi.org/10.1002/9781118303092.ch19

de Miguel, A. \& Buss, D. M. (2011). Mate retention tactics in Spain: Personality, sex differences, and relationship status. Journal of Personality, 79(3), 563-586. https://doi.org/10.1111/j.14676494.2011.00698.x

Desmarais, S. L., Reeves, K. A., Nicholls, T. L., Telford, R. P., \& Fiebert, M. S. (2012a). Prevalence of physical violence in intimate relationships, Part 1: Rates of male and female victimization. Partner abuse, $3(2)$, 140-169. https://doi.org/10.1891/1946-6560.3.2.140

Desmarais, S. L., Reeves, K. A., Nicholls, T. L., Telford, R. P., \& Fiebert, M. S. (2012b). Prevalence of physical violence in intimate relationships, Part 2: Rates of male and female perpetration. Partner Abuse, 3(2), 170-198. https://doi.org/10.1891/1946-6560.3.2.170

Easton, J. A. \& Shackelford, T. K. (2009). Morbid jealousy and sex differences in partner-directed violence. Human Nature, 20(3), 342-350. https://doi.org/10.1007/s12110-009-9069-1

Echeburúa, E. \& Amor, P. J. (2016). Hombres violentos contra la pareja:¿ tienen un trastorno mental y requieren tratamiento psicológico? Terapia psicológica, 34(1), 31-40. https://doi.org/10.4067/S071848082016000100004

Egan, V., Hughes, N., \& Palmer, E. J. (2015). Moral disengagement, the dark triad, and unethical consumer attitudes. Personality and Individual 123-128. https://doi.org/10.1016/j.paid.2014.11.054

Farvid, P., Braun, V., \& Rowney, C. (2017). 'No girl wants to be called a slut!': Women, heterosexual casual sex and the sexual double standard. Journal of Gender Studies, 26(5), 544-560. https://doi.org/10.1080/09589236.2016.1150818

Fernández-Fuertes, A. A. \& Fuertes, A. (2010). Physical and psychological aggression in dating relationships of Spanish adolescents: Motives and consequences. Child abuse \& neglect, 34(3), 183-191. https://doi.org/10.1016/j.chiabu.2010.01.002

García-Cueto, E., Rodríguez-Díaz, F. J., Bringas-Molleda, C., López-Cepero, J., Paíno-Quesada, S., \& Rodriguez-Franco, L. (2015). Development of the Gender Role Attitudes Scale (GRAS) amongst young Spanish people. International Journal of Clinical and Health Psychology, 15, 61-68. https://doi.org/10.1016/j.ijchp.2014.10.004

Graña Gómez, J. L. \& Cuenca Montesino, M. L. (2014). Prevalence of psychological and physical intimate partner aggression in Madrid (Spain): A dyadic analysis. Psicothema, 26(3), 343-348.

Hart, S. L. \& Legerstee, M. (2013). Handbook of jealousy: Theory, research, and multidisciplinary approaches. Londres: Wiley-Blackell

Hooper, D., Coughlan, J., \& Mullen, M. (2008, septiembre). Evaluating model fit: a synthesis of the structural equation modelling literature. Trabajo presentado en la séptima European Conference on research methodology for business and management studies, Londres, Reino Unido. 
Holden, C. J., Zeigler-Hill, V., Pham, M. N., \& Shackelford, T. K. (2014). Personality features and mate retention strategies: Honesty-humility and the willingness to manipulate, deceive, and exploit romantic partners. Personality and Individual 3ifferences, 57, 36. https://doi.org/10.1016/j.paid.2013.09.018

Instituto Nacional de Estadística y Censos (2019). Registro único de casos de violencia contra las mujeresRUCVM: resultados 2013-2018. Buenos Aires: Instituto Nacional de Estadística y Censos.

Jakobwitz, S. \& Egan, V. (2006). The dark triad and normal personality traits. Personality and Individual differences, 40(2), 331-339. https://doi.org/10.1016/j.paid.2005.07.006

Jonason, P. K., Li, N. P., \& Buss, D. M. (2010). The costs and benefits of the dark triad: Implications for mate poaching and mate retention tactics. Personality and Individual Differences, 48, 373-378. https://doi.org/10.1016/j.paid.2009.11.003

Jonason, P. K., Li, N. P., Webster, G. D., \& Schmitt, D. P. (2009). The Dark Triad: Facilitating a short-term mating strategy in men. European Journal of Personality, 23(1), 5-18. https://doi.org/10.1002/per.698

Jonason, P. K. \& Webster, G. D. (2010). The Dirty Dozen: A concise measure of the Dark Triad. Psychological Assessment, 22(2), 420-432. https://doi.org/10.1037/a0019265

Jones, D., \& Paulhus, D. (2011). The role of impulsivity in the Dark Triad of personality. Personality and Individual Differences, 51,670-682. https://doi.org/10.1016/j.paid.2011.04.011

Jones, D. N. \& Neria, A. L. (2015). The Dark Triad and dispositional aggression. Personality and Individual Differences, 86, 360-364. https://doi.org/10.1016/j.paid.2015.06.021

Jones, D. N. \& Paulhus, D. L. (2014). Introducing the short dark triad (SD3) a brief measure of dark personality traits. Assessment, 21(1), 28-41. https://doi.org/10.1177/1073191113514105

Jones, D. N. \& Paulhus, D. L. (2017). Duplicity among the dark triad: Three faces of deceit. Journal of Personality and Social Psychology, 113(2), 329. https://doi.org/10.1037/pspp0000139

Kaighobadi, F. \& Shackelford, T. K. (2008). Female attractiveness mediates the relationship between in-pair copulation frequency and men's mate retention behaviors. Personality and Individual Differences, 45(4), 293-295. https://doi.org/10.1016/j.paid.2008.04.013

Kaighobadi, F., Shackelford, T. K., \& Goetz, A. T. (2009). From mate retention to murder: Evolutionary psychological perspectives on men's partner-directed violence. Review of General Psychology, 13(4), 327334. https://doi.org/10.1037/a0017254

Kar, H. L. \& O'Leary, K. D. (2013). Patterns of psychological aggression, dominance, and jealousy within marriage. Journal of Family Violence, 28(2), 109-119. https://doi.org/10.1007/s10896-012-9492-7

Karakurt, G., Whiting, K., Van Esch, C., Bolen, S. D., \& Calabrese, J. R. (2016). Couples therapy for intimate partner violence: A systematic review and meta-analysis. Journal of marital and family therapy, 42(4), 567-583. https://doi.org/10.1111/jmft.12178

Khanchandani, L. \& Durham, T. W. (2009). Jealousy during dating among female college students. College Student Journal, 43(4), 1272-1279.

Kiire, S. (2017). Psychopathy rather than Machiavellianism or narcissism facilitates intimate partner violence via fast life strategy. Personality and Individual Differences, 104, 401-406. https://doi.org/10.1016/j.paid.2016.08.043

Kline, R. B. (2015). Principles and practice of structural equation modeling. Nueva York: Guilford publications.

Langhinrichsen-Rohling, J., Misra, T. A., Selwyn, C., \& Rohling, M. L. (2012). Rates of bidirectional versus unidirectional intimate partner violence across samples, sexual orientations, and race/ethnicities: A comprehensive review. Partner Abuse, 3(2), 199-230. https://doi.org/10.1891/1946-6560.3.2.199

Marks, M. J., Young, T. M., \& Zaikman, Y. (2018). The sexual double standard in the real world. Social Psychology, 50, 67-79. https://doi.org/10.1027/1864-9335/a000362

Martínez-León, N. C., Mathes, E. W., Avendaño, B.L., Peña, J. J. \& Sierra, J. C. (2018). Psychometric Study of the Interpersonal Jealousy Scale in Colombian Samples. Revista Latinoamericana de Psicología, 5O(1), 21-30. https://doi.org/10.14349/rlp.2018.v50.n1.3

Mattingly, B. A., Whitson, D., \& Mattingly, M. J. (2012). Development of the romantic jealousy-induction scale and the motives for inducing romantic jealousy scale. Current Psychology, 3, 263-281. https://doi.org/10.1007/s12144-012-9144-3

Miller, E. \& McCaw, B. (2019). Intimate partner violence. New England Journal of Medicine, 380(9), 850-857. https://doi.org/10.1056/NEJMra1807166

Miller, J. D., Vize, C., Crowe, M. L., \& Lynam, D. R. (2019). A critical appraisal of the dark-triad literature and suggestions for moving forward. Current Directions in Psychological Science, 28(4), 353-360. https://doi.org/10.1177/0963721419838233

Ministerio de las Mujeres, Géneros y Diversidad (2020). Información estadística. Recuperado de: https://www.argentina.gob.ar/generos/linea-144/informacion-estadistica

Moyano, N., Monge, F. S., \& Sierra, J. C. (2017). Predictors of sexual aggression in adolescents: Gender dominance vs. rape supportive attitudes. The European Journal of Psychology Applied to Legal Context, 9(1), 25-31. https://doi.org/10.1016/j.ejpal.2016.06.001

Muris, P., Merckelbach, H., Otgaar, H., \& Meijer, E. (2017). The malevolent side of human nature: A metaanalysis and critical review of the literature on the dark triad (narcissism, Machiavellianism, and psychopathy). Perspectives on Psychological Science, 12(2), $183-204$. https://doi.org/10.1177/1745691616666070

Nieto Ribes, B. (2015). La triada oscura de la personalidad. Adaptación al español de los cuestionarios Dirty Dozen y Short Dark Triad (Tesis de grado). Universidad Jaume, Colombia.

Oficina de Violencia Doméstica (2020a). Informe estadístico - Primer trimestre 2020. Recuperado de http://www.ovd.gov.ar/ovd/noticias.do?method=iniciar\&idTema=114

Oficina de Violencia Doméstica (2020b). Violencia en las relaciones de pareja: mujeres afectadas. Recuperado de http://www.ovd.gov.ar/ovd/verNoticia.do?idNoticia=4378n. 
Organización de las Naciones Unidas (2020). Facts and figures: Ending violence against women. Recuperado de https://www.unwomen.org/en/what-we-do/ending-violence-against-women/facts-and-figures

Pailing, A., Boon, J., \& Egan, V. (2014). Personality, the Dark Triad and violence. Personality and Individual Differences, 67, 81-86. https://doi.org/10.1016/j.paid.2013.11.018

Paulhus, D. L., Curtis, S. R., \& Jones, D. N. (2018). Aggression as a trait: the Dark Tetrad alternative. Current opinion in psychology, 19, 88-92. https://doi.org/10.1016/j.copsyc.2017.04.007

Pfeiffer, S. M. \& Wong, P. T. (1989). Multidimensional jealousy. Journal of social and personal relationships, 6(2), 181-196. https://doi.org/10.1177/026540758900600203

Pichon, M., Treves-Kagan, S., Stern, E., Kyegombe, N., Stöckl, H., \& Buller, A. M. (2020). A Mixed-Methods Systematic Review: Infidelity, Romantic Jealousy and Intimate Partner Violence against Women. International Journal of Environmental Research and Public Health, 17(16), 5682. https://doi.org/10.3390/ijerph17165682

Raskin, R. \& Hall, C. S. (1981). The Narcissistic Personality Inventory: Alternative form reliability and further evidence of construct validity. Journal of personality assessment, 45(2), 159-162. https://doi.org/10.1207/s15327752jpa4502_10

Rauthmann, J. F. (2012). The Dark Triad and interpersonal perception: Similarities and differences in the social consequences of narcissism, Machiavellianism, and psychopathy. Social Psychological and Personality Science, 3(4), 487-496. https://doi.org/10.1177/1948550611427608

Rauthmann, J. F. \& Will, T. (2011). Proposing a multidimensional Machiavellianism conceptualization. Social Behavior and Personality: an international journal, 39(3), 391-403. https://doi.org/10.2224/sbp.2011.39.3.391

Reiss, I. L. (1960). Toward a sociology of the heterosexual love relationship. Marriage and Family Living, 22(2), 139-145. https://doi.org/10.2307/347330

Sebastián, J., Verdugo, A., \& Ortiz, B. (2014). Jealousy and violence in dating relationships: gender-related differences among a Spanish sample. The Spanish journal of psychology, 17, E94. https://doi.org/10.1017/sjp.2014.99

Sierra, J. C., Monge, F. S., Iglesias, P. S., Rodríguez, K., \& Aparicio, D. L. (2010). Propiedades psicométricas de las versiones en español de la Double Standard Scale (DDS) y de la Rape Supportive Attitude Scale (RSAS) en mujeres peruanas. Cuadernos de medicina psicosomática y psiquiatría de enlace, 95, 57-66.

Sierra, J. C., Rojas, A., Ortega, V., \& Martín Ortiz, J. D. (2007).Evaluación de actitudes sexuales machistas en universitarios: primeros datos psicométricos de las versiones españolas de la Double Standard Scale (DSS) y de la Rape Supportive AttitudeScale (RSAS). International Journal of Psychology and Psychological Therapy, 7, 41-60.

Shackelford, T. K., Goetz, A. T., Buss, D. M., Euler, H. A., \& Hoier, S. (2005). When we hurt the ones we love: Predicting violence against women from men's mate retention. Personal Relationships, 12(4), 447-463. https://doi.org/10.1111/j.1475-6811.2005.00125.x

Smith, S. G., Zhang, X., Basile, K. C., Merrick, M. T., Wang, J., Kresnow, M. J., \& Chen, J. (2018). The national intimate partner and sexual violence survey: 2015 data brief-updated release. Recuperado de https://stacks.cdc.gov/view/cdc/60893

Straus, M. A. (2006). Future research on gender symmetry in physical assaults on partners. Violence against women, 12(11), 1086-1097. https://doi.org/10.1177/1077801206293335

Straus, M. A. (2007). Conflict tactics scales. Encyclopedia of domestic violence, 190, 197

Straus, M. A., Hamby, S., Boney-McCoy, S., \& Sugarman, D. (1996). The Revised Conflict Tactics Scales: Development and preliminary psychometric data. Journal of Family Issues, 17, 283-316. https://doi.org/10.1177/019251396017003001

Straus, M. A. \& Ramirez, I. L. (2007). Gender symmetry in prevalence, severity, and chronicity of physical aggression against dating partners by university students in Mexico and USA. Aggressive Behavior, 33(4), 281-290. https://doi.org/10.1002/ab.20199

Swan, S. C., Gambone, L. J., Caldwell, J. E., Sullivan, T. P., \& Snow, D. L. (2008). A review of research on women's use of violence with male intimate partners. Violence and victims, 23(3), 301-314. https://doi.org/10.1891/0886-6708.23.3.301

Tabachnick, B. G. \& Fidell, L. S. (2014). Using multivariate statistics. Massachusetts: Allyn and Bacon/Pearson Education.

Thomson, N. D., Bozgunov, K., Psederska, E., \& Vassileva, J. (2019). Sex differences on the four-facet model of psychopathy predict physical, verbal, and indirect aggression. Aggressive behavior, 45(3), 265-274. https://doi.org/10.1002/ab.21816

Thomson, N. D., Kiehl, K. A., \& Bjork, J. M. (2019). Violence and aggression in young women: The importance of psychopathy and neurobiological function. Physiology \& behavior, 201, 130-138. https://doi.org/10.1016/j.physbeh.2018.11.043

Truman, J. L. \& Morgan, R. E. (2014). Nonfatal Domestic Violence. Washington, DC: US Department of Justice, Bureau of Justice Statistics.

Visser, B., Bay, D., Cook, G., \& Myburgh, J. (2010). Psychopathic and antisocial, but not emotionally intelligent. Personality and Individual Differences, 48, 644-648. https://doi.org/10.1016/j.paid.2010.01.003.

Wade, T. J. \& Walsh, H. (2008). Does the Big-5 relate to jealousy, or infidelity reactions? Journal of Social, Evolutionary, and Cultural Psychology, 2(3), 133. https://doi.org/10.1037/h0099349

Williams, J. R., Ghandour, R. M., \& Kub, J. E. (2008). Female perpetration of violence in heterosexual intimate relationships: Adolescence through adulthood. Trauma, Violence, \& Abuse, 9(4), 227-249. https://doi.org/10.1177/1524838008324418

Zeigler-Hill, V., Besser, A., Morag, J., \& Campbell, W. K. (2016). The Dark Triad and sexual harassment $\begin{array}{llll}\text { proclivity. Personality and Individual } & \text { 47fferences, 89, }\end{array}$ 
https://doi.org/10.1016/j.paid.2015.09.048

Recibido 21-08-2020 | Aceptado 02-02-2021 